\title{
Power Efficient Scheduled-Based Medium Access Control Protocol over Wireless Sensor Networks
}

\author{
Muhammad Shoaib Khan, Abrar Alajlan, Marwah Almasri \\ Department of Computer Science and Engineering, University of Bridgeport, Bridgeport, USA \\ Email: mukhan@my.bridgeport.edu, aalajlan@my.bridgeport.edu,maalmasr@my.bridgeport.edu
}

Received 9 September 2014; accepted 22 February 2016; published 25 February 2016

Copyright (C) 2016 by authors and Scientific Research Publishing Inc.

This work is licensed under the Creative Commons Attribution International License (CC BY). http://creativecommons.org/licenses/by/4.0/

(c) (i) Open Access

\begin{abstract}
The flexible use of sensors has made them an attractive device to be deployed in almost every field of life such as health, military and home. Recent advancement in electronics and wireless communications has witnessed the development of low cost-sensor devices. While wireless sensor networks (WSNs) are flexible to use and less costly, they need to be more energy-efficient as they are operated by the battery. Mostly they are deployed in harsh environments where it is very difficult to change the batteries frequently. Several medium access control (MAC) algorithms have been developed for the energy-efficient acquisition of the wireless channel, however, none of them are satisfactory. In this paper, we proposed a medium access control algorithm called MAC-PE. MACPE is based on the concept of prioritized frames where prioritized frames are transmitted urgently. In addition, it uses scheduled-based MAC instead of accessing channel randomly. We found MAC-PE (Power-Efficient MAC) was efficient in terms of power consumption without sacrificing on the performance using NS-2.
\end{abstract}

\section{Keywords}

PE-MAC, S-MAC, G-MAC, T-MAC, Power Consumption, Mobility

\section{Introduction}

Wireless Sensor Networks are rapidly growing research area in recent years due to its significant applications in many disciplines such as healthcare, surveillance, military, and homeland security. However, sensors have limited power sources. For this reason, particular attention is needed to manage the energy consumption efficiently at each node. 
Today, power consumption of wireless sensor networks is a very challenging issue given that each sensor node is equipped by a limited power source due to the hardware constraints. Moreover, power consumption in WSNs can be utilized by tree functions, that is, sensing, processing, and communication. Given the fact, most energy in WSNs is consumed by the communication among nodes. Therefore, it is really important to have very efficient communication protocols for the lifetime of WSNs. Wireless channel is a sharing media, that is, each sensor node shares the same media. Therefore, there is necessary to have an efficient Medium Access Control protocol (MAC).

Medium Access Control (MAC) protocol has a significant role in maintaining the wireless channel in WSNs. Communications between nodes cost sufficient energy, which eventually degrades the network. Hence, a power efficient MAC protocol in WSNs increases the efficiency of the network and thus prolonging the network lifetime.

Several MAC protocols have been introduced to handle overhearing avoidance, idle listening and message passing [1].

Another MAC protocol is T-MAC, which is based on S-MAC. It uses an adaptive duty cycle algorithm based on network loads. It dynamically selects duty cycle and decreases the idle listening while keeping enough throughput simultaneously. In order to adjust the duty cycle, T-MAC uses a strategy called ADS-MAC. Depending on recent periods, this approach employs statistical calculations for throughput to estimate the throughput at future periods [2].

In addition, the queuing system of the MAC protocol influences the network performance. Having high load traffic will eventually overflow the buffer queue of the node, and thus the packets need to be either discarded or retransmitted which will consume more energy [3].

In this paper, we propose a power efficient scheduled-based MAC protocol for Wireless Sensor Networks. This protocol employs two types of queues which are: Priority-based Queue and Normal Queue. In the Priority-based Queue, priority traffic is stored which is determined based on the significance of traffic. Priority is set in the packet by using the one extra flag bit that shows the priority of the packet. This protocol reduces the energy consumption, the number of control packets, and end-to-end delay. In addition, it increases the number of delivered packets and the lifetime of the network.

The remainder of the paper is organized as follows: Section 2 presents the related work; Section 3 outlines system model and assumptions; Section 4 describes our proposed algorithm, i.e., PE-MAC; in Section 5 we analyze the results obtained from the simulations; Section 6 discusses the results and finally entire paper is concluded in Section 7.

\section{Related Work}

This section discusses some of the various existing MAC protocols in the literature that concern with the power consumption dilemma especially in the WSNs. Others are focusing on reducing the transmission costs and delay. A new asynchronous MAC protocol with QoS awareness (AQ-MAC) in WSN has been proposed by [4]. This protocol allows the receiver to initiate the transmission. Packets are classifiedas ahigh priority or low priority packets. The high priority packets are sent right away where the low priority packets saved in a queue waiting for other high priority packets to arrive or until a timeout. This protocol has been evaluated by NS2 at various scenarios as it reduces delays and saves a lot of energy. In addition, authors in [5] presented a mathematical model for the cognitive radio networks (CRNs), where using a priority-based MAC protocol. Primary users have higher priorities than the secondary users that result in reducing the processing and the transmission time for all users.

In addition, a novel queuing analytical model for distributed and energy-aware MAC protocol with service differentiation has presented by [6]. In each node, the O-MAC layer works as a server where a vacation queuing model has been used to control sleep/awake mode. They also considered the limitation of energy and buffered size by using high and low priority traffics. Many performance indicators have been used to analyze the efficiency of the model such as packet dropping probability, throughput, queue length distribution, energy consumption, and access delay.

In addition, authors in [7] proposed an energy efficient priority-based QOS MAC protocol for WSNs (PRIMA). This protocol first groups node into clusters and then uses both TDMA and CSMA model for the channel access phase. CSMA is employed for control messages while TDMA is used for data messages in order to reduce collisions. In addition, it uses a queuing model that is consists of four different queues to categorize the packets according to its importance as high or low priority packets. PRIMA protocol has been evaluated 
through OMNet++ modular and showed that it has a better performance than Q-MAC protocol. It has lower energy consumption, lower average packet delay, and higher average delivery ratio compared to Q-MAC protocol.

In [8], authors presented a queuing analytical model of polling based wireless MAC protocols in WSNs. They studied the performance of polling based MAC in terms of buffer overflow rates, packet delay, and energy consumption. In addition, a distributed packet-based cross-layer streaming algorithm thatis based on priority queuing in a multi-hop wireless network has been analyzed by [9]. This algorithm takes into account the priority queuing in order to transmit the video packet that is assignedto a high priority packet over the most reliable link. In addition, this algorithm has proved to have a better network scalability as it adapts to network changes and high-quality performance.

In [10], authors have improved the S-MAC protocol by merging two techniques which are adaptive bakeoff window and estimating the data flow based on the queue's length. This protocol is called R-MAC. Based on NS2.34 simulator, R-MAC minimized latency and power consumption that are primary factors to prolong the WSNs lifetime. Another approach dealing with the queue load based on traffic priority for bandwidth sharing in MAC protocol is introduced by [11]. This ensures the QOS in WSNs. Authors have discussed the effect of queue load on bandwidth where the loading rate has a great impact on traffic priority and channel access.

In addition, QA-MAC protocol, which is based on S-MAC protocol, is proposed by [12]. QA-MAC employs the mechanism of adjusting the contention window size based on queue traffic. It outperforms the S-MAC in terms of energy saving by $28.7 \%$ that significantly enhances the performance of WSNs.

Another MAC protocol is called CH-MAC protocol that uses both CSMA and TDMA mechanisms and a real queuing system. The queuing system is a fair share queuing system that means all packets are dropped evenly so no particular node will be affected. On the other hand, the drop-tail queuing system consumes a lot of energy and degrades the network performance. CH-MAC protocol has been evaluated based on power consumption, packet delivery ratio, and latency [13]. However, these protocols work in static situation [14], but degrade the performance in mobile scenario. In this paper, we focus on both static and mobile scenarios.

\section{System Model and Assumptions}

Mobile ad hoc system is functional to numerous conditions with no use of any accessible system. Military situations necessitate the network to route data packet throughout energetically mobile nodes (Figure 1). MANETs considered as the answer for this highly mobile and dynamic military system. It is not suitable to directly relate conventional mobile ad hoc networks system to military network systems, as military communication network is



Figure 1. Realistic military communication system. 
unlike from conventional both in physical layer condition and the networking atmosphere. First considering these specifications of military communication networks to out imitation, and assess the performance of three ad hoc routing algorithms on the unspecified military situations. The easiness of operation with no accessible communications makes ad hoc system a gorgeous option for dynamic condition such as military operations. Particularly, military situation considered as the unique incentive intended for MANET, owing to the requirement for battleground survivability and rapid operation of mobile communications. Modern research in ad hoc network systems focused on medium access control (MAC) and routing, ensuing in numerous protocols intended and various quantitative analysis work compares the performance of these protocols.

\section{Proposed Methodology for PE-MAC}

Our proposed power efficient protocol aims to save the power. The nodes in PE-MAC initially attempt to sense the carrier to transmit and receive the data. When a node obtains the slot then waits for specific time to send the data in order to let other nodes to transmit respective packets. The packet forwarded by node is assigned the priority based on the nature of the packets. The next node checks the priority of the packet if packet is marked as priority level then packets are forwarded to the next node based on the priority and keeps going this process until the packets reach to the destination node. In case, packets are not assigned the priority then will be stored into buffer for specific time until all of the priority- based packets are forwarded. In our scheme three levels of priority is set and packets are treated according to priority. Figure 2 shows the entire process of getting the channel and forwarding the packets.

The end-to-end delay is critical when forwarding the packets to the next node because throughput of any approach depends on the end-to-delay. Algorithm 1 presents the process for determining the end-to-end delay for forward traffic.

In this algorithm, the queues are initialized; firstly critical queues and normalized queues .Traffic is also initialized as priority-based traffic and then normal traffic. Initialization of parameters like delay time, propagation time, transmission time, end-to-end delay, processing time at each node, number of hops and overhead of

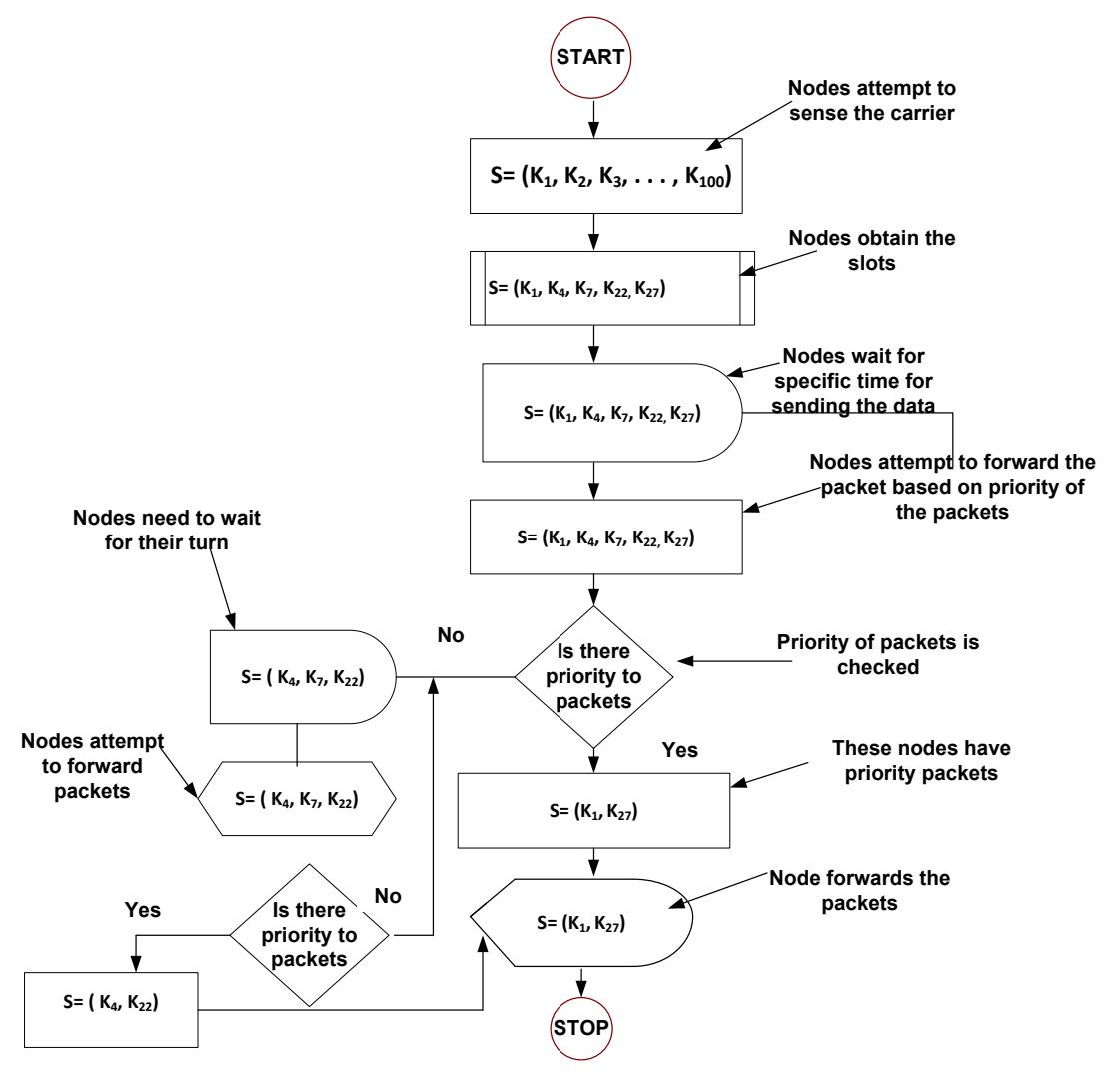

Figure 2. Channel access and data forwarding process based on priority of traffic. 
Algorithm 1. Queue selection and end-to-end delay for different traffics.

1. Initialization of queues $\left(\Delta C_{q}\right.$ : Critical Queue, $\Delta N_{q}$ : Normal Queue)

2. Initialization of traffic ( $T$ : Traffic, $T_{p}$ : priority-based traffic $\& T_{n}$ : Normal traffic)

3. Initialization of parameters $\left(T_{d}\right.$ : Delaytime, $P_{t}$ : Propagation time, $\frac{D_{p}}{T_{s}}$ : Transmission time of data into medium, $D_{e}$ : End-to-end delay,

$T_{p t}$ : Processing time of $T_{p}$ at each node, $H$ : Number of hops \& $O_{h}$ : Overhead of each hop

4. While node " $K$ " send " $T$ " to node " $K_{1}$ " at cluster " $L$ "

5. If $T=T_{p}$ then

6. Node $K_{1}$, putting $T_{p}$ into $\Delta C_{q}$

7. Else if $T=T_{n}$ then

8. Node $K_{1}$, putting $T_{n}$ into $\Delta N_{q}$

9. End if

10. End if

11. Examining delay time " $T_{d}$ " for $\Delta C_{q}$

12. Do $\frac{T_{d}}{T_{p}}$ // Transmitting data from source to destination

13. If $\frac{T_{d}}{T_{p}} \in \Delta C_{q}$, then

14. Calculate $D_{e}=N \times \frac{D_{p}}{T_{s}}+T_{p t}+\frac{T_{d}}{T_{p}}+\left(N \times O_{h}\right)$ // End-to-End delay for priority-based traffic

15. Else if $\frac{T_{d}}{T_{p}} \in \Delta N_{q}$, then

16. Calculate $D_{e}=N \times \frac{D_{p}}{T_{s}}+N_{q_{\text {dday }}}+T_{p}+\frac{T_{d}}{T_{p}}+\left(N \times O_{h}\right) / / \quad N_{q_{-} \text {delay }}$ : Additional delay for normal queue \& $\Delta T_{p t}:$ Additional processing time at each node; End-to-End delay for normal traffic

17. End if

18. End if

19. End while

each hop. While, node is executed at the cluster to pass the values from one node to the other. Then if else statements are executed to decide the time; if it equals to the priority-based traffic then the code assign pro- cessing time in the critical queue at node $\mathrm{K} 1$ else to normal traffic and assign this to normal queue.

The code examines delay time for critical. The code calculates delay time, transmission time of data and transmits data from source to destination. If statement is executed, ration of delay time to processing time equates critical. Then End-to-End delay for priority-based traffic is calculated. Else if the ratio equates normal queue then End-to-End delay for normal traffic is intended.

The residual energy of the node demonstrates the lifetime of the network. Hence, Algorithm 2 shows the listening process and node life.

\section{Algorithm 2. Determining the idle listening process and remaining node life.}

1. Variable Initialization ( $K$ : Sensor node; $\Delta t$ : Task; $\mu$ : Idle state; $S_{t}$ : Synchronization time; $I_{s}:$ Idle state; $\Delta t_{c}$ : Total tasks $\& O_{s}$ : Off state)

2. Input: ( $E_{\text {init }}:$ Initial energy of sensor node; $E_{\text {idle }}:$ Consumed energy in idle state)

3. Output: ( $S_{l}:$ Sensor node lifetime)

4. For $\left(\Delta t=0 ; \Delta t \leq \Delta t_{c} ; \Delta t+1\right)$

5. Compute $\Delta t$

6. If $\mathrm{K}$ completes $\Delta t$ then

7. $K=\mu / /$ Sensor node goes to idle state temporarily to check its next task

8. $K \in S_{t}$ // Sensor node checks its next synchronization time

9. Swamping $O_{s} \leftarrow I_{s} / /$ Sensor node goes from idle state to sleep state

10. Else if $K \neq \mu \quad / /$ Sensor node needs to continue task

11. End if

12. End if

13. Calculate $S_{l}=\frac{E_{\text {init }}}{E_{\text {idle }}} \quad / /$ Lifetime of sensor node based on energy

14. For end 
Algorithm 2 is responsible for determining the idle listening process and remaining node life, and it also consists of three phases, that is, Variable Initialization, Processing, and In Variable Initialization phase, the algorithm initializes the following variable: sensor node $[K]$, task, idle state $[\mu]$, synchronization time, idle state, total tasks, and off state. In processing phase, the algorithm takes initial energy of sensor node and consumed energy in idle state as inputs, then the algorithm initializes a loop counter, task $=0$, and increases the value of the counter, task +1 , to compute task until the condition is satisfied, task should be less than or equal total task. If the sensor node $[K]$ completes task, then the sensor node $[K]$ goes to idle state temporarily to check its next task, then initializing a loop counter to a value and increasing or decreasing the value for certain no of times until a condition is satisfied.

\section{Simulation Setup and Analysis of Result}

In order to determine the performance of our proposed PE-MAC in the wireless sensor network, we implemented our approach using NS2 [15] with 13.10 Ubuntu Operating system. Network is designed covering $500 \times$ 500 square meters. We distributed 100 nodes in the network with homogenous capabilities. Each node has initial 10 joules energy. The objective of this simulation is to determine the power efficiency to improve the QOS. Furthermore, we also compared our approach with other three known existing protocols: An adaptive energy-efficient MAC protocol (T-MAC) [16], medium-access control (MAC) protocol designed for wireless sensor networks(S-MAC) [17], and Gateway MAC (G-MAC) [18].

We demonstrated two scenarios, with and without mobility. The simulation scenarios are consists of 12 end nodes, which create flat topology. We set medium access layer to operate with the non-beacon enabled mode. Radio range of each sensor nodes under free-space propagation model is fixed to 30 meters. The distance between each sensor node is set 35 meters. In this section we will analyze the results obtained after performing several experiments. Table 1 shows various simulation parameters used for the experiments.

Figure 3 shows the energy consumption in Joules by our MAC-PE and compares it with T-MAC, S-MAC and G-MAC. The result shows that our proposed algorithm consumes less energy as compared to other protocols. In this experiment we kept the sensor nodes static MAC-PE consumes $25 \%$ less than S-MAC when the number of sensor nodes is 100 . It also consumes $13 \%$ and $12 \%$ less energy than T-MAC and G-MAC.

Figure 4 shows energy consumption comparison of our proposed algorithm with T-MAC, S-MAC and

Table 1. Showing simulation parameters.

\begin{tabular}{cc}
\hline Name of parameters & Description \\
\hline Transmission range & 30 meters \\
Sensing range of node & 20 meters \\
Initial energy of a node & 10 Joules \\
Bandwidth of node & $40 \mathrm{~Kb} / \mathrm{Sec}$ \\
Number of sensors & 100 \\
Size of network & $500 \times 500$ square meters \\
Buffering capacity & 50 Packets at each node \\
Data Packet size & 128 bytes \\
Simulation time & 450 seconds \\
Initial pause time & 05 Seconds \\
Type of Queues & Priority and Normal \\
$T_{x}$ energy & 14 mW \\
$R_{x}$ energy & 13 mW \\
MAC protocol & 0.0005 seconds \\
Node Wait time in Slot & MACrient (MA-PE) \\
\hline
\end{tabular}




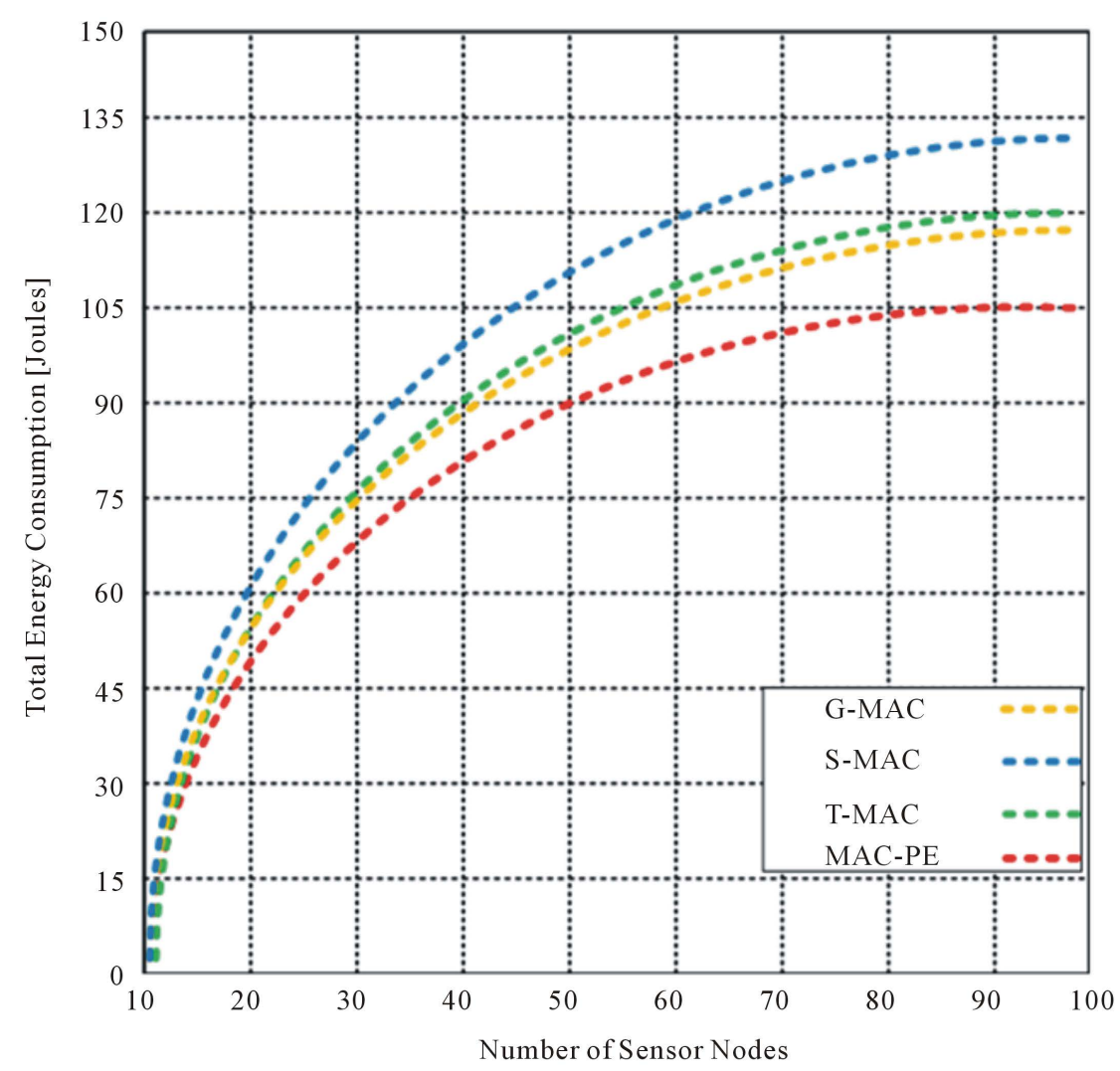

Figure 3. Total energy consumption with static nodes.

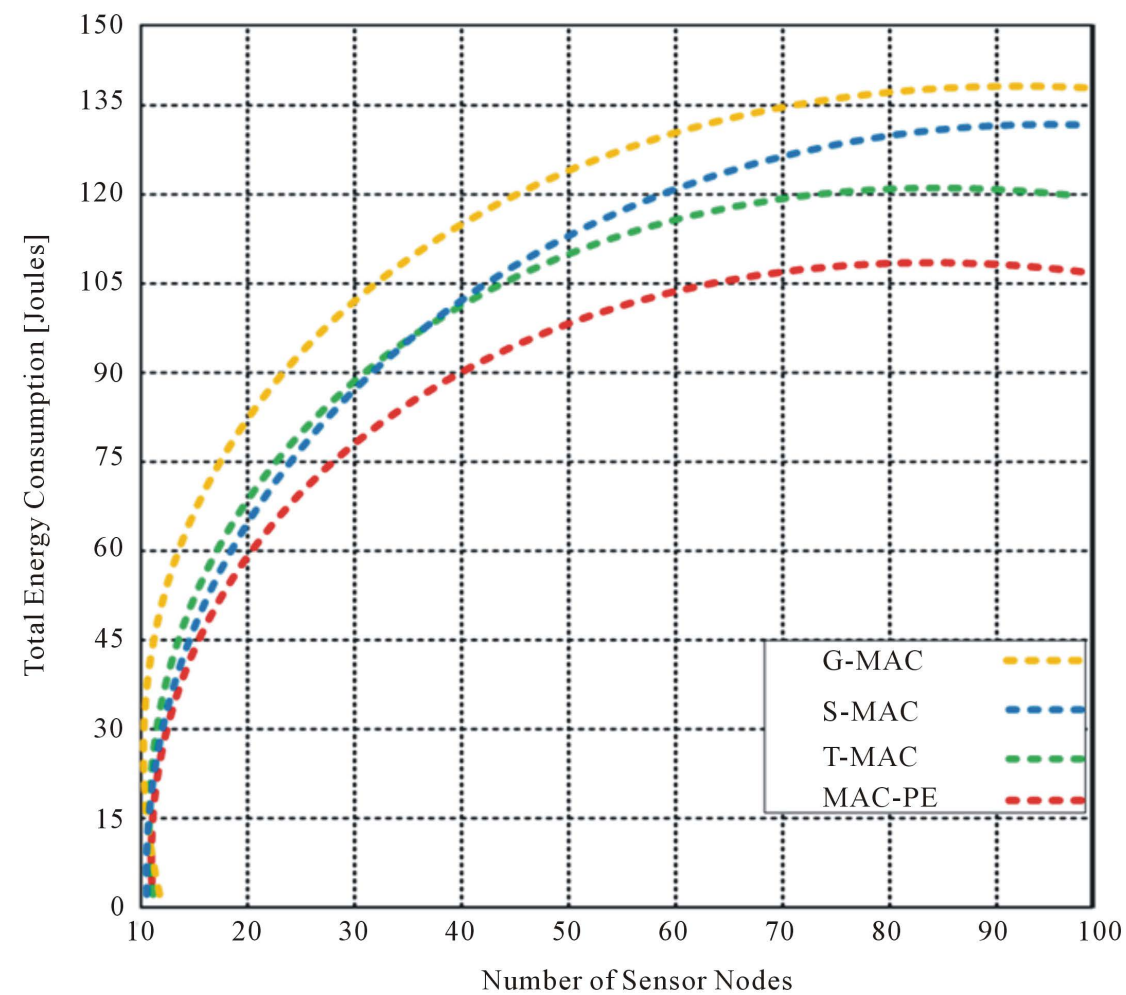

Figure 4. Total energy consumption with $50 \%$ moving nodes. 


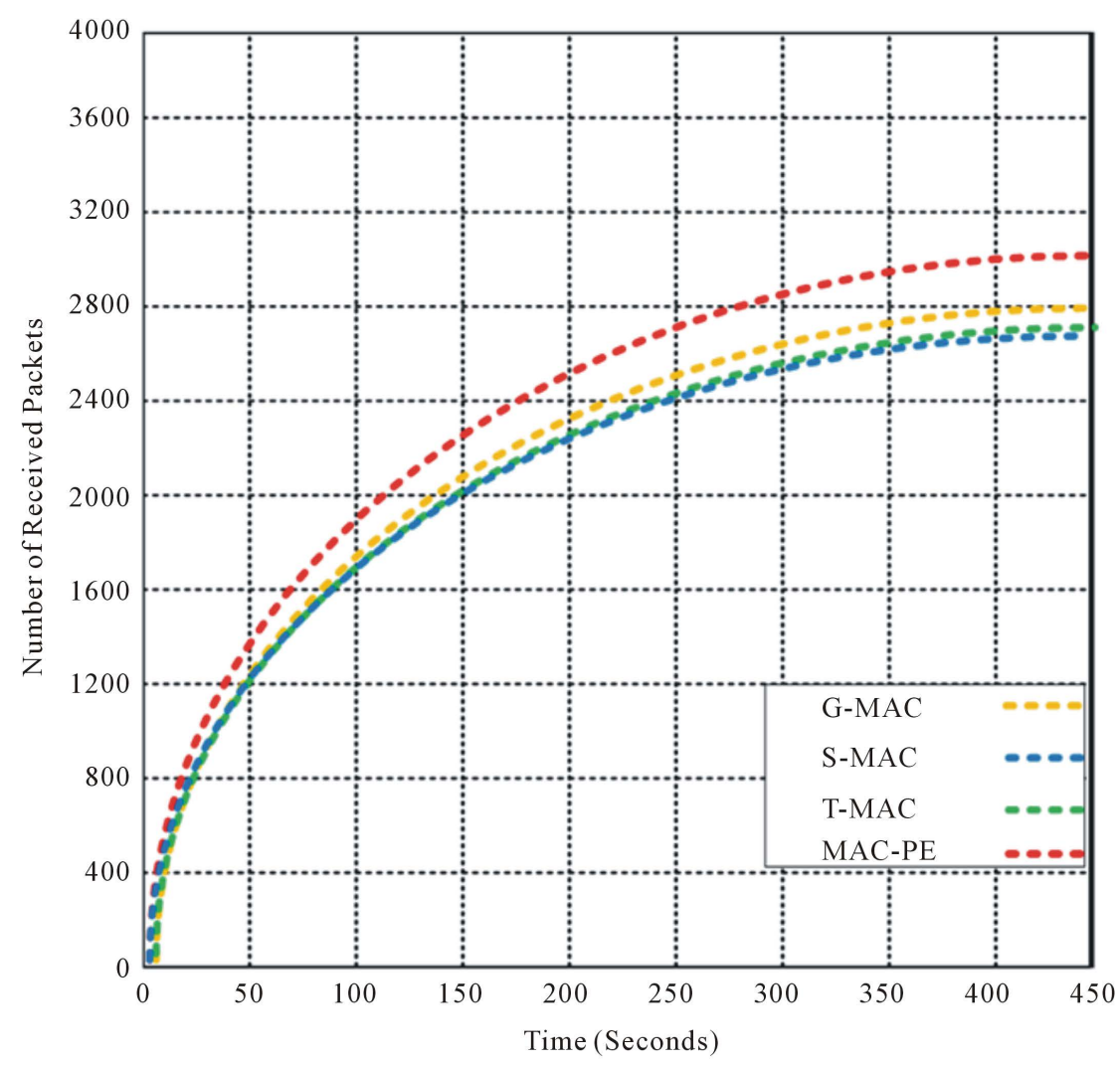

Figure 5. Number of delivered packets with static nodes.

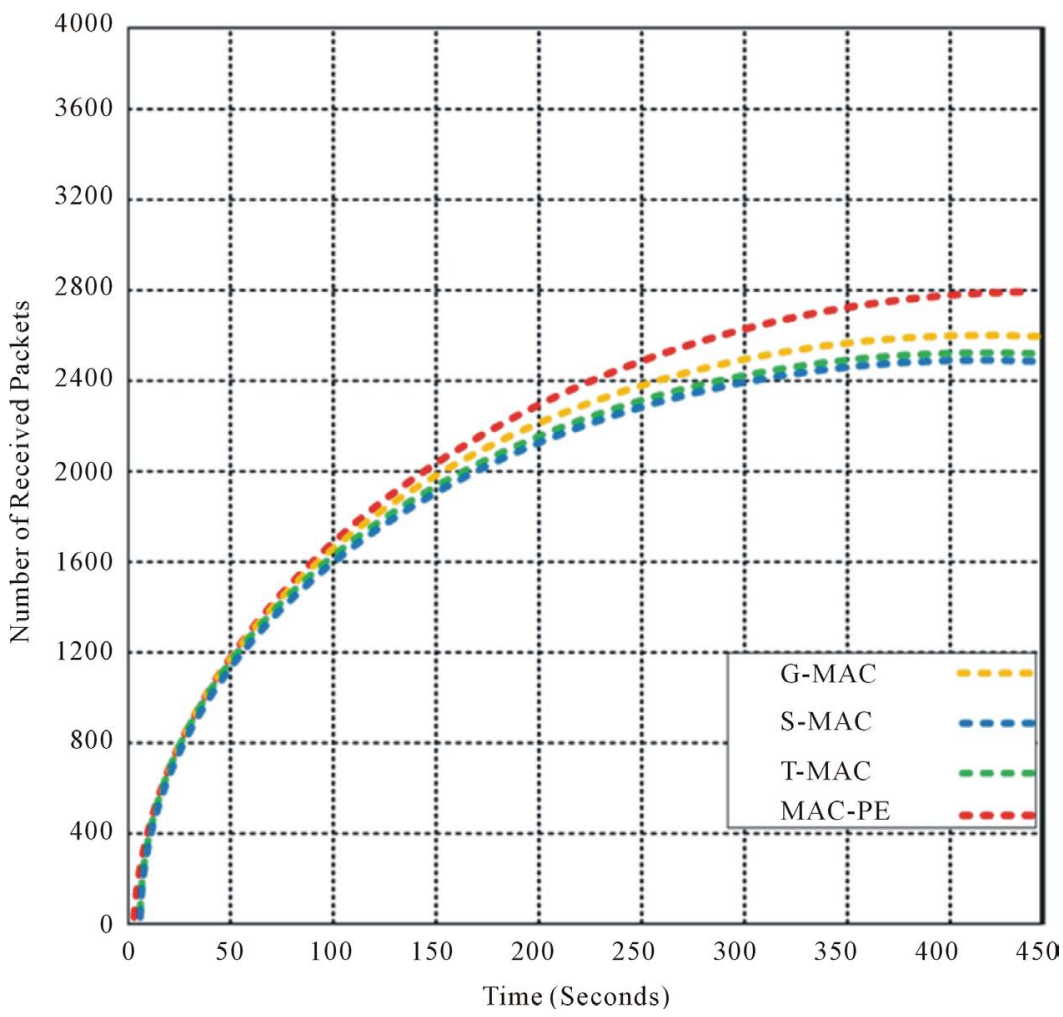

Figure 6. Number of delivered packets with 50\% moving nodes. 


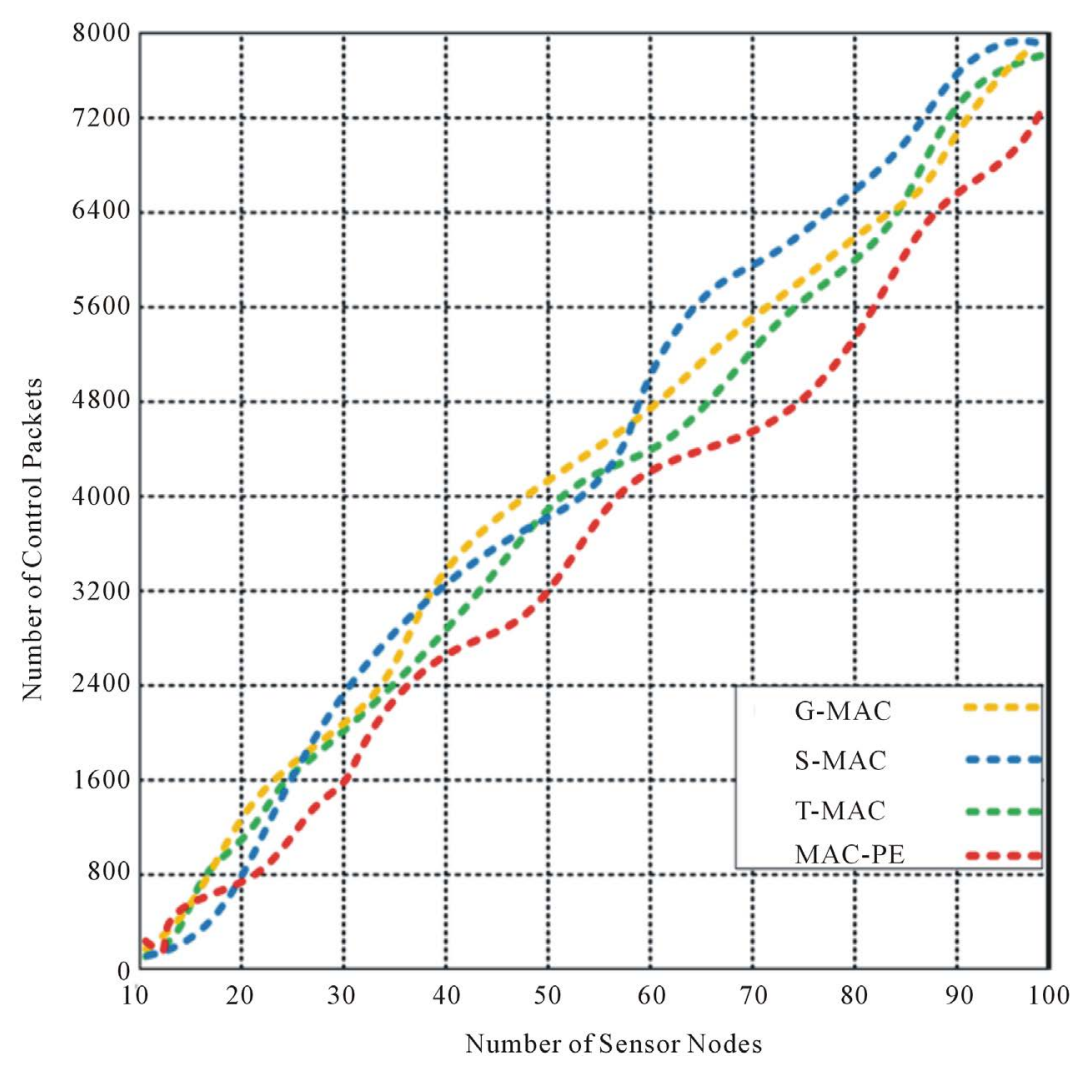

Figure 7. Number of control packets with static nodes.

G-MAC. In this experiment half of sensor nodes present in network are moving whereas the other half are set static. It is found that MAC-PE consumes almost $28 \%, 24 \%$ and $13 \%$ less energy than G-MAC, S-MAC and T-MAC, respectively.

Figure 5 shows the number of successfully received packets. All sensor nodes in network are kept static. It can be seen from the result that MAC-PE receives highest number of packets as compared to the other three protocols, followed by G-MAC, T-MAC and S-MAC.

Figure 6 shows the result for the number of packets successfully received in sensor. In this experiment, half of the sensor nodes present in network are in the moving state. When mobility increases that PE-MAC and competing MAC protocols decrease the pefromance. However, our PE-MAC is less affected when number of sendor nodes increase.

Figure 7 shows the number of control frames transmitted/received successfully in sensor network of 100 nodes. It shows that our proposed PE-MAC protocol uses the least number of control frames. The highest number of frames is used by S-MAC followed by G-MAC and T-MAC. The higher the number of control frames transmitted the higher the energy consumption by the network.

\section{Discussion of Results}

MAC-PE reveals itself as a promising alternative to existing MAC protocols for WSNs, i.e. S-MAC, T-MAC and G-MAC. It's more efficient than these protocols even when half of sensor nodes present in the network are moving.

Our evaluation shows that our proposed protocol reduces the energy consumption and extends the network lifetime accordingly. This fact is due to several features. On one hand, our protocol reduces the idle state which leads to conserve energy. On the other hand, it allows to avoid the buffer queue overflow by introducing two types of queues which are Priority-based Queue for prioritized traffic and Normal Queue for normal traffic. This feature allows to avoid energy waste caused by retransmissions. MAC-PE reduces also end-to-end delay since prioritized frames are transmitted urgently without additional delay in the queue of each node. Furthermore, MAC- 
PE enhances network reliability since it uses scheduled-based MAC instead of random channel access which reduces collisions and packet loss accordingly.

\section{Conclusion}

In this paper we presented our proposed algorithm MAC-PE. Performance of MAC-PE is evaluated in terms of energy consumption, end-to-end delay and lifetime of sensor nodes. We compared the performance of our proposed algorithm with three other protocols, i.e., G-MAC, S-MAC and T-MAC. It was proved by simulations that our proposed algorithm out-performs all the three protocols in terms of power consumption and throughput when all the nodes are static or half of the nodes are mobile. Our proposed PE-MAC is validated using NS2 and results confirm that our PE-MAC could be used for improving the network life and quality of service for different wireless sensor networks. In future, we use our PE-MAC in large scale wireless sensor networks and will particularly focus on the multiple applications.

\section{References}

[1] Ye, W., Heidemann, J. and Estrin, D. (2004) Medium Access Control with Coordinated Adaptive Sleeping for Wireless Sensor Networks. IEEE/ACM Transactions on Networking, 12, 493-506.

[2] Razaque, A. and Elleithy, K.M. (2014) Energy-Efficient Boarder Node Medium Access Control Protocol for Wireless Sensor Networks. Sensors, 14, 5074-5117. http://dx.doi.org/10.3390/s140305074

[3] Razaque, A. and Elleithy, K. (2015) Nomenclature of Medium Access Control Protocol over Wireless Sensor Networks. IETE Technical Review, 1, 1-2. http://dx.doi.org/10.1080/02564602.2015.1057769

[4] Nguyen, K. and Ji, Y. (2012) Asynchronous MAC Protocol with QOS Awareness in Wireless Sensor Networks. IEEE Global Communications Conference (GLOBECOM), Anaheim, 3-7 December 2012, 555-559. http://dx.doi.org/10.1109/glocom.2012.6503171

[5] Chouhan, L. and Trivedi, A. (2012) Priority Based MAC Scheme for Cognitive Radio Network: A Queuing Theory Modelling. 2012 9th International Conference on Wireless and Optical Communications Networks (WOCN), Indore, 20-22 September 2012, 1-5. http://dx.doi.org/10.1109/wocn.2012.6331886

[6] Razaque, A. and Elleithy, K. (2015) Modular Energy-Efficient and Robust Paradigms for a Disaster-Recovery Process over Wireless Sensor Networks. Sensors, 15, 16162-16195. http://dx.doi.org/10.3390/s150716162

[7] Ben-Othman, J., Mokdad, L. and Yahya, B. (2011) An Energy Efficient Priority-Based QOS MAC Protocol for Wireless Sensor Networks. IEEE International Conference on Communications (ICC), Kyoto, 5-9 June 2011, 1-6. http://dx.doi.org/10.1109/icc.2011.5962414

[8] Yang, H. and Sikdar, B. (2012) Queuing Analysis of Polling Based Wireless MAC Protocols with Sleep-Wake Cycles. IEEE Transactions on Communications, 60, 2427-2433.

[9] Shiang, H. and Schaar, M. (2007) Multi-User Video Streaming over Multi-Hop Wireless Networks: A Distributed, Cross-Layer Approach Based on Priority Queuing. IEEE Journal on Selected Areas in Communications, 25, $770-785$.

[10] Lin, Q., Chuan, D.H. and Jiao, X. (2012) Study of Medium Access Control Protocol Based on Adaptive Back off Window in Wireless Sensor Network. International Symposium on Information Technology in Medicine and Education (ITME), 2, 885-888.

[11] Lagkas, T., Angelidis, P., Stratogiannis, D. and Tsiropoulos, G. (2010) Analysis of Queue Load Effect on Channel Access Prioritization in Wireless Sensor Networks. 6th IEEE International Conference on Distributed Computing in Sensor Systems Workshops (DCOSSW), Santa Barbara, 21-23 June 2010, 1-6. http://dx.doi.org/10.1109/dcossw.2010.5593273

[12] Gao, L. (2011) A Energy Consumption Improvements of S-MAC in WSN. International Conference on Internet Technology and Applications (ITAP), Wuhan, 16-18 August 2011, 1-3. http://dx.doi.org/10.1109/itap.2011.6006172

[13] Priya, B. and Manohar, S. (2013) CH-MAC: Congestion Control Hybrid MAC for Wireless Sensor Network. 4th International Conference on Computing, Communications and Networking Technologies (ICCCNT), Tiruchengode, 4-6 July 2013, 1-6. http://dx.doi.org/10.1109/iccent.2013.6726686

[14] Rizvi, S., Karpinski, K. and Razaque, A. (2015) Novel Architecture of Self-Organized Mobile Wireless Sensor Networks. Journal of Computing Science and Engineering, 9, 163-176. http://dx.doi.org/10.5626/JCSE.2015.9.4.163

[15] Razaque, A. and Elleithy, K.M. (2014) Low Duty Cycle, Energy-Efficient and Mobility-Based Boarder Node-MAC Hybrid Protocol for Wireless Sensor Networks. Journal of Signal Processing Systems, 81, 265-284.

[16] Van Dam, T. and Langendoen, K. (2003) An Adaptive Energy-Efficient MAC Protocol for Wireless Sensor Networks. 
Proceedings of the 1st International Conference on Embedded Networked Sensor Systems, New York, 5 November 2003, 171-180. http://dx.doi.org/10.1145/958491.958512

[17] Ye, W., Heidemann, J. and Estrin, D. (2002) An Energy-Efficient MAC Protocol for Wireless Sensor Networks. Proceedings of IEEE 21st Annual Joint Conference of the IEEE Computer and Communications Societies, 3, 1567-1576.

[18] Brownfield, M.I., Mehrjoo, K., Fayez, A.S. and Davis IV, N.J. (2006) Wireless Sensor Network Energy-Adaptive Mac Protocol. 3rd IEEE Consumer Communications and Networking Conference (CCNC), 2, 778-782. 